\title{
Combined Effect of Magnetic field and Internal Heat Generation on the Onset of Marangoni Convection
}

\author{
Gangadharaiah \\ Department of Mathematics, Sir M Visvesvaraya Institute of Technology, Bangalore, India
}

Email address:

Gangu.honnappa@gmail.com

To cite this article:

Gangadharaia. Combined Effect of Magnetic field and Internal Heat Generation on the Onset of Marangoni Convection. International

Journal of Fluid Mechanics \& Thermal Sciences. Vol. 3, No. 4, 2017, pp. 41-45. doi: 10.11648/j.ijfmts.20170304.12

Received: April 21, 2017; Accepted: September 15, 2017; Published: October 23, 2017

\begin{abstract}
Marangoni convection in a horizontal layer with a uniform internal heat source and vertical magnetic field is analyzed. The boundaries are considered to be rigid, however permeable, and insulated to temperature perturbations. The upper surface of a fluid layer is deformably free. The eigen value equations of the perturbed state obtained from the normal mode analysis are solved by using regular perturbation method with $a$ as wave number. The results show that the critical Marangoni number $M_{c}$ become larger as the Chandrasekhar number $Q$ increases, internal heat source and the Crispation number $\mathrm{Cr}$ decreases.
\end{abstract}

Keywords: Internal Heat Source, Magnetic Field, Marangoni Convection

\section{Introduction}

The analysis of Benard-Marangoni convection in a thin fluid layer induced by thermal buoyancy and surface tension is important for many applications in science and engineering. Examples include energy storage in molten salts, crystal growth from a melt in space, and paints, colloids and detergents in chemical engineering. Nield [1] initiated the study of the Benard-Marangoni convective instability in a planar horizontal fluid layer with a non-deformable free surface. Later, Davis and Homsy [2] extended the work of Nield [1] to take the effect of the surface deflection into account. When the Crispation effect of a deformably free upper surface is considered, oscillatory instabilities may occur in the Benard-Marangoni problem. Takashima, M [3] presented a detailed numerical study of the linear stability analysis of Benard-Marangoni convection, including stationary and oscillatory modes, and focused the influence of the Crispation number on the conditions for a competition between two of these kinds of modes. Char and Chiang [4] examined the boundary effects on the Benard-Marangoni instability problem in the presence of an electric field, and found that the boundary effects of the solid plate have great influences on the stability of the system. Recently, Hashim and Wilson [5] advanced the analyses of [3,4] to the Benard-
Marangoni instability of a horizontal liquid layer in the most physically-relevant case when Rayleigh number and Marangoni number are linearly dependent. All these previous investigators are restricted to the convective system in the absence of a magnetic field. Chandrasekhar [6] studied the Rayleigh-Benard convective instability induced by buoyancy in a magnetic field. The onset of steady Marangoni instability in an electrically conducting fluid layer with a nondeformable free surface in a magnetic field was first considered by Nield [1]. He found that the Lorentz force is to contract Marangoni convection. Later, Maekawa and Tanasawa [7] extended the analysis of [1] to investigate the effect of orientation of the magnetic field and the aspect ratio of the liquid layer on the onset of Marangoni convection.

The effect of quadratic basic state temperature gradient caused by uniform internal heat generation plays a decisive role in understanding control of convection. Copious literature is available on coupled Benard-Marangoni convection in a horizontal ordinary viscous fluid layer with uniform distribution of internal heat generation (Char and Chiang [4], Wilson [8], Bachok and Arifin [9] and references therein). The problem of penetrative convection in an ordinary viscous fluid-saturated porous layer has also received considerable attention in the recent past because of its applications in many science and engineering problems (with current highly relevant literature including Carr [10], 
Chang ([11-13]), Gangadharaiah and Suma [14], Gangadharaiah ([15-18]).

In the present study, we have considered the problem of combined buoyancy and surface tension driven convection in a horizontal fluid layer in the presence of uniform vertical magnetic field including the additional effect of internal heat generation.. The lower rigid and upper free boundary at which the temperature-dependent surface tension forces are accounted for are considered to be perfectly insulated to temperature perturbations. The resulting eigenvalue problem is solved by regular perturbation technique with wave number as a perturbation parameter.

\section{Mathematical Formulation}

We consider penetrative convection via internal heating in a system consisting of an infinite horizontal fluid layer of thickness $d$ and the z-axis pointing vertically upwards opposing the direction of gravity. The temperatures of the lower and upper boundaries are taken to be uniform and equal to $T_{l}$ and $T_{u}$ respectively, with $T_{l}>T_{u}$. The upper free surface of fluid layer is free of deformities with its position being $z=d+\Omega(x, y, t)$.

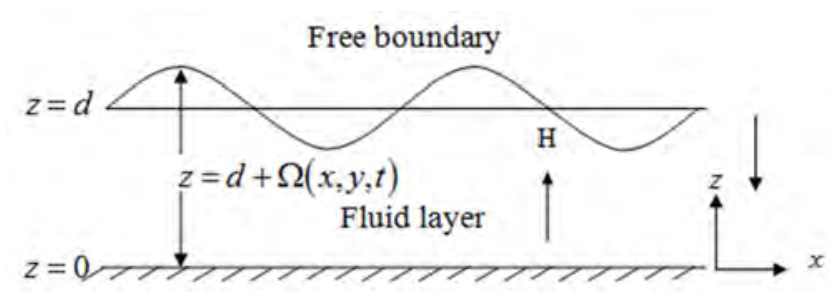

Figure 1. Physical Configuration.

The governing equations for the fluid layer are:

$$
\nabla \cdot \vec{V}=0
$$

$$
\begin{gathered}
\rho_{0}\left(\frac{\partial \vec{V}}{\partial t}+(\vec{V} \cdot \nabla) \vec{V}\right)=-\nabla p+\mu \nabla^{2} \vec{V} \\
\frac{\partial T}{\partial t}+(\vec{V} \cdot \nabla) T=k \nabla^{2} T+q \\
\nabla \cdot \vec{H}=0 \\
\frac{\partial \vec{H}}{\partial t}+(\vec{V} \cdot \nabla) \vec{H}=(\vec{H} \cdot \nabla) \vec{V}+\mu \nabla^{2} \vec{H}
\end{gathered}
$$

In the above equations, $\vec{V}=(u, v, w)$ is the velocity vector, $p$ is the pressure, $T$ is the temperature, $q$ is the heat source in the fluid layer, $\kappa$ is the thermal diffusivity and $\rho_{0}$ is the reference fluid density.

The basic state is quiescent and is of the form

$$
(u, v, w, p, T, H)=\left[0,0, W_{0}, p_{b}(z), T_{b}(z), H_{b}(z)\right]
$$

The basic steady state is assumed to be quiescent and temperature distributions are found to be

$$
\begin{aligned}
& T_{b}(z)=T_{0}-\left[\left(\frac{\left(T_{0}-T_{u}\right)}{d}-\frac{q d}{2 \kappa}\right) z+\frac{q}{2 \kappa} z^{2}\right] \\
& \vec{H}_{b}(z)=\left[H_{0}-\left(\left(\frac{\Delta T}{d}-\frac{q d}{2 \kappa}\right) z+\frac{q}{2 \kappa} z^{2}\right)\right] \hat{k}
\end{aligned}
$$

Where $T_{0}$ is the interface temperature. In order to investigate the stability of the basic solution, infinitesimal disturbances are introduced in the form

$$
\vec{V}=\vec{V}^{\prime}, \quad T=T_{b}+T^{\prime}, \quad p=p_{b}+p^{\prime}, \quad \rho=\rho_{b}+\rho^{\prime}, \quad \mu=\mu_{b}+\mu^{\prime}
$$

where the primed quantities are the perturbations and assumed to be small. Eq.(9) is substituted in Eqs. (1)-(5) and linearized in the usual manner. The pressure term is eliminated from Eq. (2) by taking curl twice on these two equations and only the vertical component is retained. The variables are then non-dimensionalized using $d, d^{2} / \kappa, \kappa / d$ and $T_{0}-T_{u}$ as the units of length, time, velocity, and temperature in the fluid layer and Then performing a normal mode expansion of the dependent variables. we obtain the following ordinary differential equations

$$
\begin{array}{r}
\left(D^{2}-a^{2}\right)^{2} W-Q D^{2} W=0 \\
\left(D^{2}-a^{2}\right) \Theta=-W[1-N s(1-2 z)]
\end{array}
$$

$$
\operatorname{Pr}_{m}\left(D^{2}-a^{2}\right) \psi=-D W
$$

The linearized boundary conditions are:

$$
\begin{gathered}
W=D \psi=D \Theta+B i[\Theta-(1+N s) Z]=0 \quad \text { at } \quad z=1 \\
\left(D^{2}+a^{2}\right) W+M a^{2}[\Theta-(1+N s) Z]=0 \quad \text { at } z=1 \\
C r\left[\left(D^{2}-3 a^{2}\right) D W-Q\left(D^{2}-a^{2}\right) \psi\right]=\left(B_{0}+a^{2}\right) a^{2} Z \quad \text { at } z=1 \\
W=0, D W=0 \text { and } D \Theta=0=D \psi \quad \text { at } z=0 .
\end{gathered}
$$

\section{Method of Solution}

Since the critical wave number is exceedingly small for the assumed temperature boundary conditions (Nield and Bejan 
2006) the eigen value problem is solved using a regular perturbation technique with wave number $a$ as a perturbation parameter. Accordingly, the dependent variables are expanded in powers of $a^{2}$ in the form

$$
(W, \Theta, \psi)=\sum_{i=0}^{N}\left(a^{2}\right)^{i}\left(W_{i}, \Theta_{i}, \psi_{i}\right)
$$

Substitution of Eq. (17) into Eqs. (10)-(12) and the boundary conditions $(13)-(16)$

$$
\begin{gathered}
D^{4} W_{0}-Q D^{2} W_{0}=0 \\
D^{2} \theta_{0}=-f(z) W_{0} \\
D^{2} \psi_{0}=-D W_{0}
\end{gathered}
$$

where

$$
f(z)=[1-N s(1-2 z)]
$$

The boundary conditions (13)-(16) become

$$
\begin{gathered}
W_{0}=D W_{0}=D \Theta_{0}=0 \quad \text { at } z=0 \\
D^{2} W_{0}=D \Theta_{0}=0 \quad \text { at } z=1
\end{gathered}
$$

Then solutions to above equations are

$$
W_{0}=0 \text { and } \Theta_{0}=1
$$

First- order equations $(10)-(12)$ become

$$
\begin{gathered}
D^{4} W_{1}-Q D^{2} W_{1}=0 \\
D^{2} \Theta_{1}=1-f(z) W_{1} .
\end{gathered}
$$

The boundary conditions $(13)-(16)$ become

$$
\begin{gathered}
W_{1}=D W_{1}=0 \quad \text { at } z=0 \\
D^{2} W_{0}+M a^{2}[\Theta-(1+N s) Z]=0 \quad \text { at } z=1 \\
D^{3} W_{1}-\frac{B_{0}}{C r} Z_{0}=0 \text { at } z=1
\end{gathered}
$$

The general solution of $(25)$ is

$$
W_{1}=C_{1}+C_{2} z+C_{3} e^{-\sqrt{Q} z}+C_{4} z e^{\sqrt{Q} z}
$$

Where $C_{1}, C_{2}, C_{3}$ and $C_{4}$ are constants and they have to determined using the appropriate boundary conditions.

$$
\begin{aligned}
& C_{1}=-\frac{e^{-Q / 2}}{2 Q^{2}}\left(\frac{e^{-Q}+Q-7}{1-2 e^{-Q}-Q^{2} e^{-Q}}\right), C_{2}=\frac{e^{-Q / 2}}{-2 Q^{2}}\left(\frac{2-2 e^{Q}+Q}{e^{2 Q}-Q^{2} e^{Q}-1}\right) \\
& C_{3}=\frac{e^{Q / 2}}{2 Q^{2}}\left(\frac{e^{Q}-Q-1}{1+e^{2 Q}-2 e^{Q}-Q^{2} e^{Q}}\right), C_{4}=\left(\frac{2-2 e^{Q}+2 Q+Q^{2}}{2 e^{Q}-e^{2 Q}+Q^{2} e^{Q}-1}\right) .
\end{aligned}
$$

The differential Equation (26) involving $D^{2} \Theta_{1}$ provide the $\quad M=48$. solvability requirement which is given by

$$
\int_{0}^{1} f(z) W_{1} d z=1
$$

The expressions for $W_{1}$ is back substituted into Eq. (31) and integrated to get Marangoni number

$$
\frac{M}{\Delta_{1}+\Delta_{2}}=1
$$

Where $\Delta_{1}=-\frac{e^{-Q / 2}}{2 Q^{2}}\left(\frac{e^{-Q}-Q-1}{1-2 e^{-Q}+e^{-2 Q}-Q^{2} e^{-Q}}\right)$,

$\Delta_{2}=\frac{e^{-Q}-Q-1}{\left(\mathrm{Q}^{2}-\mathrm{Cr}(1+N s)\right)+(2 \mathrm{Q}-3 \operatorname{Cr}(1+N s)+6 \mathrm{Q}}$

In the limit absence of internal heating (i.e., $N s \rightarrow 0$ ) and magnetic field (i.e., $Q \rightarrow 0$ ), we recover the know result

\section{Results and Discussion}

The effect of internal heat generation on the criterion for the onset of Marangoni instability in the presence of vertical magnetic field with upper surface of a fluid layer is deformably free is investigated theoretically. The resulting eigen value problem is solved using a regular perturbation technique with wave number $a$ as a perturbation parameter.

The presence of internal heating makes the basic temperature and magnetic field distributions to deviate from linear to parabolic in terms of the porous layer height which in turn has significant influence on the control of Marangoni convection. To assess the impact of internal heat source strength on the stability of the system, the dimensionless basic temperature $\hat{T}_{b}(z)$, and magnetic field intensity $\hat{H}_{b}(z)$ distributions are exhibited graphically in Figure 2 for different values of internal heat source strength NS. From the figure, it is observed that an increase in the internal heat 
source strength amounts to large deviations in these distributions which in turn enhance the disturbances within the medium and thus reinforce instability on the system.

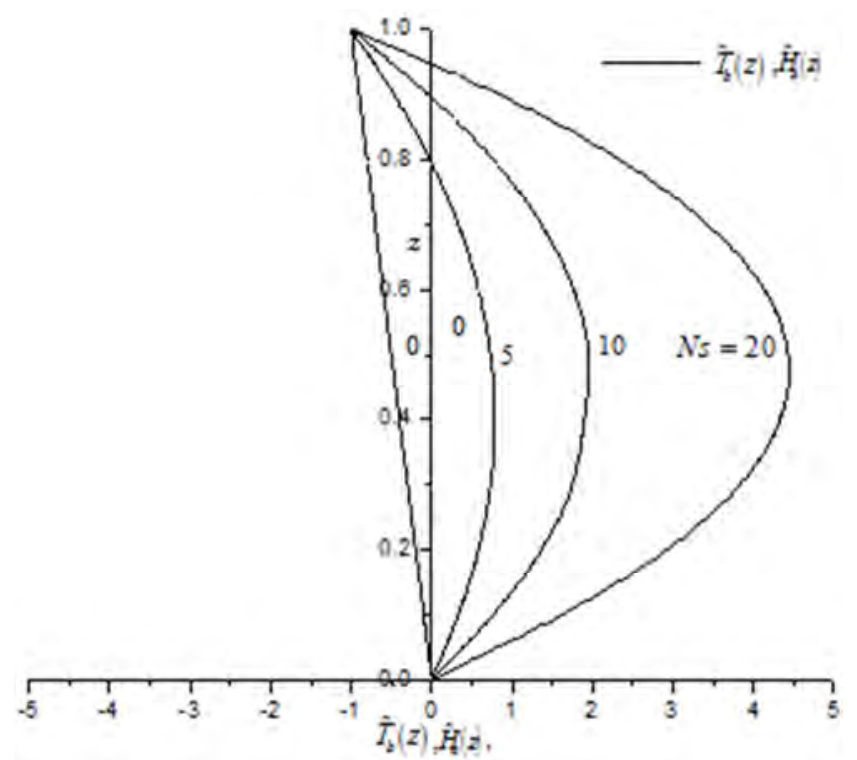

Figure 2. Basic State Temperature Distributions for Different Values of Ns .

Figure 3 depicts the critical Marangoni number $M_{c}$ as a function of the Chandrasekhar number Q for different values of Ns. It is found that the critical Marangoni number increases as the values of the Chandrasekhar number Q and decreases as with increasing internal heat source strength. With increasing $\mathrm{Q}$, the strength of the magnetic field increases and thus the intensity of Marangoni convection is reduced. $\mathrm{NS}$

Figure 4 depicts the critical Marangoni number $M_{c}$ as a function of internal heat source $N s$ for different values of $Q$. It is found that the critical Marangoni number decreases as the values of the heat source strength $N s$ increases and Chandrasekhar number Q and decreases. With increasing Q, the strength of the magnetic field increases and thus the intensity of Marangoni convection is reduced.

The effect of Crispation number on the critical Marangoni numbers for different values of Chandrasekhar number $Q$ is plotted in Figure 5. From Figure 5, it can be seen that in the absence of magnetic field $(\mathrm{Q}=0)$ the critical Marangoni number $M_{c}$ decreases as $\mathrm{Cr}$ increases. This effect is attributed to the fact that a higher value of $\mathrm{Cr}$, representing a lower rigidity of the free upper surface of the fluid layer, makes the system more unstable. In addition, the dotted lines in the figure indicated the critical Marangoni number corresponding to an imposed magnetic field with $Q=4.0$. An inspection of the figure reveals that the critical Marangoni number is higher in the presence of magnetic field when compared with the case of no applied magnetic field. This indicates that the applied magnetic field reduces the intensity of Marangoni convection and thus leads to a more stable system.

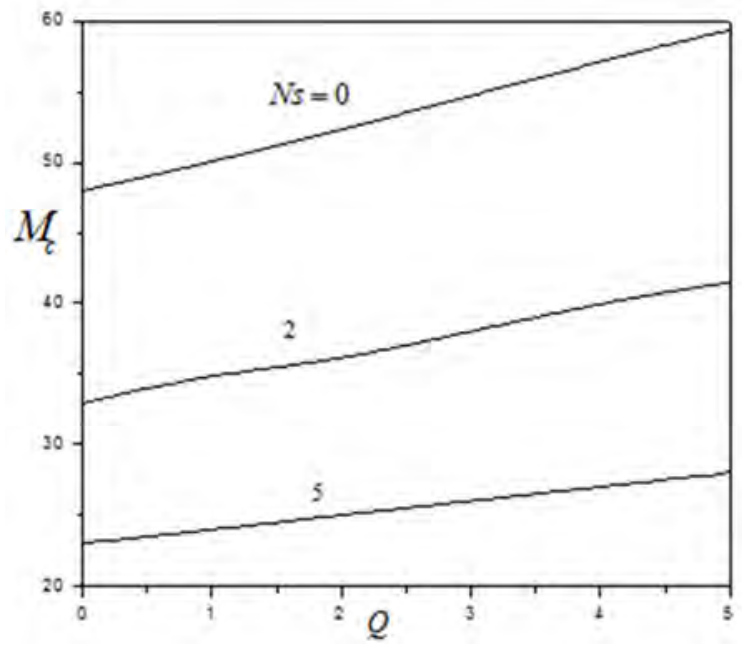

Figure 3. The Effect of $Q$ on the Stability of Marangoni Convection for Different Values of Ns with $B_{0}=0.1, R=0$ and $C r=10^{-5}$.

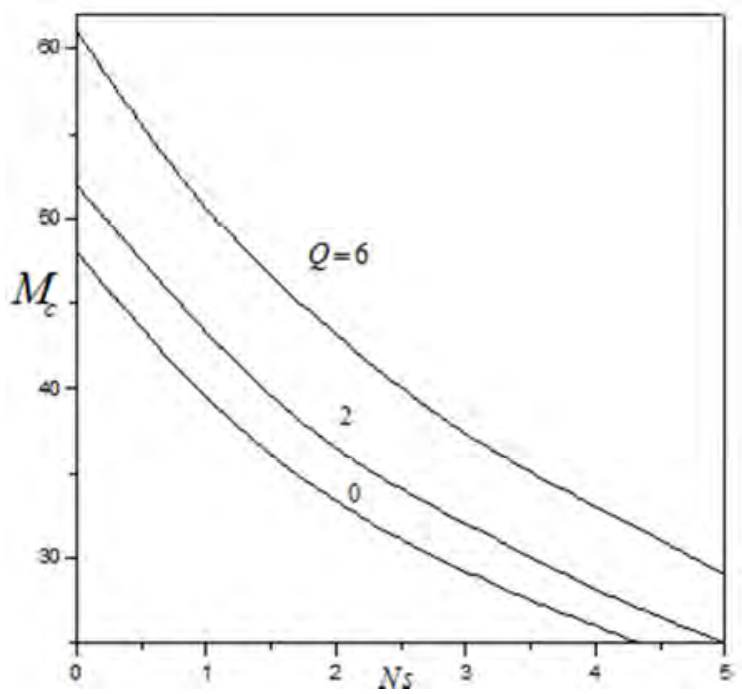

Figure 4. The Effect of Ns on the Stability of Marangoni Convection for Different Values of $Q$ with $B_{0}=0.1, R=0$ and $C r=10^{-5}$.

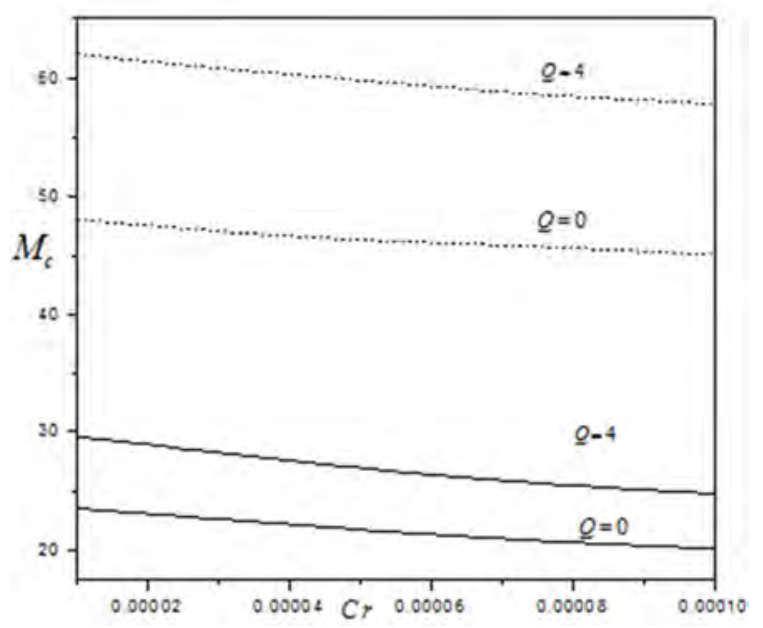

Fiureg 5. The Effect of $\mathrm{Cr}$ on the Stability of Marangoni Convection for Different Values of $Q$ with $B_{0}=0.1, R=0$; Solid Line, Ns $=5$; Dotted Line, Ns $=0$. 


\section{Conclusions}

The problem of Marangoni convection in an electrically conducting fluid layer permeated by a uniform, vertical magnetic field has been studied theoretically. Of interest are the influences of internal heat source, imposed magnetic field, and the Crispation on the onset of Marangoni instability. The following conclusions may be made from this study.

1. When the layer is heated from below, the critical Marangoni number decrease monotonically with the increase internal hear source strength.

2. The effects of the Chandrasekhar number on the onset of Marangoni convections are more pronounced, especially for the fluid layer.

3. The critical Marangoni number increase as the Chandrasekhar number increases and decreases with the increasing Crispation number.

\section{References}

[1] Nield, D. A., "Surface tension and buoyancy effect in cellular motion", J. Fluid Mech. vol.19, pp. 341-348, 1964.

[2] S. H. Davis, and G. M. Homsy., "Energy stability theory for free surface problems: buoyancy-thermocapillary layers", J. Fluid Mech, vol. 98, pp. 527-553, 1980.

[3] Takashima, M., "Surface tension driven instability in a horizontal liquid layer with a deformable free surface. I. Stationary convection", Journal of the Physical Society of Japan. Vol. 50, pp. 2745-2750, 1981.

[4] Char, M. I., Chen, C. C., "Effect of non-uniform basic temperature gradients on the onset of oscillatory Marangoni convection in a magnetic field", Acta Mech. Vol.161, pp. 17$30,2003$.

[5] Hashim, I., Wilson, S. K., "The onset of Benard -Marangoni convection in a horizontal layer of fluid", Int. J. Engg, Sci, vol.37, pp. 643, 1999.

[6] S. Chandrasekhar., "Hydrodynamic and Hydromagnetic Stability", oxford at the Clarendon Press, 1961.
[7] Maekava, T., Tanasuwa, I., "Effect of magnetic field on convection", Int. J. Heat Mass Transfer, vol. 34, pp. 285 , 1988.

[8] Wilson, S. K., "The effect of uniform internal heat generation on the onset of steady Marangoni convection in a horizontal layer of fluid", Acta Mech. Vol. 124, pp. 63-69, 1997.

[9] Bachok, N., Arifin, N. M., "Feedback control of the Marangoni-Benard convection in a horizontal fluid layer with internal heat generation", Microgravity Sci. Technol. vol. 22, pp.97-105.2010.

[10] M. I. Char, K. T. Chiang., "Stability analysis of BenardMarangoni convection in fluids with internal heat generation", J. Phys. D, vol. 27, pp. 748-755. 1964.

[11] Carr, M., "Penetrative convection in a superposed porousmedium-fluid layer via internal heating", J. Fluid Mech. Vol. 509, pp. 305-329, 2004.

[12] Chang, M. H., "Stability of convection induced by selective absorption of radiation in a fluid overlying a porous layer", Phys. Fluids. Vol. 16, pp. 3690-3698, 2004.

[13] Chang, M. H., "Thermal convection in superposed fluid and porous layers subjected to a horizontal plane couette flow", Phys. Fluids. Vol. 17, pp. 1-7. 2005.

[14] Chang, M. H., "Thermal convection in superposed fluid and porous layers subjected to a plane Poiseuille flow", Phys. Fluids. Vol. 18, pp. 3-10, 2006.

[15] Gangadharaiah. Y. H., "Bernard-Marangoni Convection in a Fluid layer Overlying a Layer of an Anisotropic Porous Layer with Deformable Free surface", Journal of Applied Fluid Mechanics, vol. 9, pp. 221-229, 2016.

[16] Gangadharaiah. Y. H., "Onset of Benard-Marangoni Convection in a Composite Layers with Anisotropic Porous Material", Journal of Applied Fluid Mechanics, vol. 10, pp. 661-66, 2017.

[17] Gangadharaiah. Y. H, (2017): Onset of Darcy-Benard Penetrative Convection in Porous Media. Journal of Applied Fluid Mechanics, 10, 661-666,.

[18] Gangadharaiah Y. H., "Double-Diffusive Marangoni convection in a composite system". International Journal of Innovative Research in Science, Engineering and Technology. 131, 137-144, 2013. 